\title{
The effect of diet composition and multiplicity on weight, body fat and HOMA-IR in patients with metabolic syndrome.
}

\author{
$\underline{\text { Nataliia Samoilenko }}^{1}$, Vira Khorunzha ${ }^{2,1}$, Olga Bezugla ${ }^{1}$, Karina Deineko ${ }^{1}$, Marta Lisevych $^{1}$ and \\ Ganna Bielokoz \\ ${ }^{1}$ Dietology clinic of Samoilenko, Kyiv, Ukraine and \\ ${ }^{2}$ National medical University, Kyiv, Ukraine
}

\begin{abstract}
Introduction: Many studies have shown relationships between timing of food intake and insulin sensitivity. In addition to this, composition of food could be an important contributor to disruptions of the normal metabolism. The aim of this study was to assess the effect of diet composition and multiplicity on weight, body fat and insulin resistance in patients with metabolic syndrome (MS).
\end{abstract}

Materials and Methods: We tested 56 patients with MS (25 men and 31 women) who were 19 to 61 years of age. Patients were on 3-fold meal (food intake was at 8-9 a.m., 1-2 p.m. and 6-7 p.m.) with very low-carbohydrate (LC) breakfast and supper, lunch consisted of proteines (P), LC, polyunsaturated fatty acids (PUFAs) and non-starchy vegetables(V); there were no snacks during a day; diet intervension lasted 6 weeks. No drugs were used during this period. Body composition analysis was performed by bioimpedance analyzer (Omron BF511, Japan). Homeostatic model assessment-insulin resistance (HOMA-IR) was used to index IR. Self-reported diet records were used to assess dietary intake. Statistical analysis was based on using pair t-test with assessment of the dynamics of clinical indicators with 95\% CI and calculation of the standardized effect size based on mean comparison (Cohen's d).

Results: 46 patients $(82,1 \%)$ completed the trial, physical activity didn't change substantially during this period. After 6-weeks BMI had reduced from $30,95 \pm 5,31 \mathrm{~kg} / \mathrm{m}^{2}$ to $29,14 \pm 5,13 \mathrm{~kg} / \mathrm{m}^{2}$ (treatment effect $-1,81 ; 95 \% \mathrm{CI}-2,25$ to $-1,37, \mathrm{p}<0,001$ ). Mean body fat was $39,4 \pm 7,87 \%$ at baseline, and $36,08 \pm 8,72 \%$ after 6 weeks of diet, treatment effect $-3,32 ; 95 \% \mathrm{CI}-4,34$ to $-2,29$, p < 0,001 treatment effect was mostly observed among men $(-4,9)$ than in women $(-2,4)$. The most significant changes were observed in HOMA-IR score - it decreased from 4,03 $\pm 2,21$ to $2,28 \pm 1,37$, treatment effect $-1,74 ; 95 \% \mathrm{CI}-2,29$ to $-1,21, \mathrm{p}<0,001-$ treatment effect was also mostly observed among men $(-2,46)$ than in women $(-1,25)$.

Discussion: Our findings demonstrate that this diet associated with statistically different changes in BMI, body fat and HOMA-IR. Individuals with obesity, particularly men, turned to group of overweight (BMI $\left.<30 \mathrm{~kg} / \mathrm{m}^{2}\right)$. Maximum effet size was in HOMA-IR changes - in 35 patients $(76,1 \%)$ there were no insulin resistance markers after 6 week of diet intervention. So, 3-fold meal with very LC breakfast and supper, mixed lunch ( LC $+\mathrm{P}+\mathrm{PUFAs}+\mathrm{V})$, without any snacks could be good addition or even alternative to pharmacological treatment of MS.

\section{Conflict of Interest}

There is no conflict of interest 